\title{
A Stochastic Predator-Prey Model
}

\author{
RANDALL J. SWIFT
}

\begin{abstract}
A stochastic version of the classical predatorprey population model is considered through a simple birthdeath formulation. The expected value of the process is obtained and shown to correspond to the deterministic predatorprey population model.
\end{abstract}

\section{INTRODUCTION}

Often in courses in probability theory and mathematical modeling, simple birth-death processes are studied which provide a useful introduction to stochastic modeling. The birth, death and birth-death processes are well known, and elementary treatments of these processes can be found in the recent probability text [3] or the modeling text [2].

These processes are natural stochastic generalizations of the deterministic population model for population growth of a single species inhabiting an environment in which the amount of resources never changed and the number of other species also remained fixed.

In this note, we will consider a natural stochastic generalization of the classical predator-prey model. The predator-prey model was originally proposed by A. J. Lotka and V. Volterra in the 1920's. It is a relatively simple model to formulate and it is often studied in elementary differential equation courses. The classical model is formulated by considering two interacting populations at a time $t$, a predator species, denoted $f(t)$ and a prey species, $r(t)$.

2000 Mathematics Subject Classification. 60J80, 92A20.

Key words and phrases. Predator-Prey population model, birth-death process. 
In the absence of predators, the prey species exhibit exponential growth. In particular,

$$
\frac{d r(t)}{d t}=\alpha r(t),
$$

where $r(t)>0$ for all $t$ and $\alpha$ is the prey birth rate. It is assumed that there is always sufficient food for the prey. Similarly, in the absence of prey, the predators die exponentially. That is,

$$
\frac{d f(t)}{d t}=-\delta f(t)
$$

where $f(t)>0$ for all $t$ and $\delta>0$ is the predator death rate. It can be assumed there is other food for the predators, but it is not sufficient to sustain the population.

When both populations are present, the interaction of the species is modeled as a product of the population sizes (i.e. $r(t) f(t)$ ). The term $r(t) f(t)$ is borrowed from chemistry models of rates of reaction where molecules in solution interact by randomly bumping into one another. An interesting discussion of this interaction term and the classical predator-prey model can be found in the recent modeling text [2].

In the classical model, a predator-prey interaction results in a prey death at a rate $\beta r(t) f(t)$, where $\beta>0$. Since it is assumed that the prey are the primary food source for the predators, the predators have a birth rate $\gamma r(t) f(t)$, where $\gamma>0$.

The classical predator-prey model, with the assumptions discussed above, yields the following system of equations:

$$
\begin{aligned}
& \frac{d r(t)}{d t}=\alpha r(t)-\beta r(t) f(t) \\
& \frac{d f(t)}{d t}=\gamma r(t) f(t)-\delta f(t),
\end{aligned}
$$

where $\alpha, \beta, \gamma, \delta$ are all nonnegative constants. In the next section, a stochastic version of this model is considered.

\section{A Stochastic Predator-Prey Model}

Let $R(t)$ be the size of the prey population at time $t$ and $F(t)$ be the size of the predator population at time $t$. In the model to be formulated, it is now assumed that instead of a (deterministic) rate of predator and prey births and deaths, there is a probability of a predator and prey birth or death. Thus $R(t)$ and $F(t)$ are time dependent random variables. 
Let the probability of there being $r$ preys and $f$ predators at time $t$ be denoted by

$P_{r, f}(t)=P[R(t)=r, F(t)=f]$, for $r=0,1,2, \ldots, f=0,1,2, \ldots$.

As in the simple birth-death process for a single species, births and deaths in this process occur proportional to the population size. We assume that the infinitesimal probability of an individual prey birth during the small time interval $\Delta t$ is $\alpha r \Delta t+o(\Delta t)$, where $\alpha>0$ is the prey birth rate. Similarly, we assume that the infinitesimal probability of an individual predator death during the small time interval $\Delta t$ is $\delta f \Delta t+o(\Delta t)$, where $\delta>0$ is the predator death rate.

To mimic the deterministic model interaction term, we assume that the infinitesimal probability of a prey death occuring during $\Delta t$ is $\beta r f \Delta t+o(\Delta t)$, where $\beta>0$ is the prey death rate. Similarly, a predator birth occurs during $\Delta t$ with infinitesimal probability $\gamma r f \Delta t+o(\Delta t)$, with $\gamma>0$.

Thus, this predator-prey process can be described as having the following transitions and rates:

$$
\begin{array}{cc}
\text { transition } & \text { rate } \\
r \rightarrow r+1, f \rightarrow f & \alpha r \\
r \rightarrow r-1, f \rightarrow f & \beta r f \\
r \rightarrow r, f \rightarrow f+1 & \gamma r f \\
r \rightarrow r, f \rightarrow f-1 & \delta f
\end{array}
$$

The standard argument using the forward Kolmogorov equations is used to obtain $P_{r, f}(t)$, by considering the probability $P_{r, f}(t+\Delta t)$. This probability is obtained as the sum of the probabilities of the following mutually exclusive events:

a) There are $r$ prey and $f$ predators by time $t$ and no birth or deaths of either species occur in $(t, t+\Delta t)$.

b) There are $r-1$ prey and $f$ predators by time $t$ and a prey birth occurs in $(t, t+\Delta t)$.

c) There are $r$ prey and $f-1$ predators by time $t$ and a predator birth occurs in $(t, t+\Delta t)$.

d) There are $r+1$ prey and $f$ predators by time $t$ and one prey death occurs in $(t, t+\Delta t)$.

e) There are $r$ prey and $f+1$ predators and one predator death occurs in $(t, t+\Delta t)$. 
It is assumed that $\Delta t$ is sufficiently small to guarantee that only one such event can occur in $(t, t+\Delta t)$. These probabilities yield

$$
\begin{aligned}
P_{r, f}(t+\Delta t)= & (1-\beta r f+\delta f+r \alpha+r f \gamma+o(\Delta t)) \Delta t P_{r, f}(t) \\
& +(\alpha(r-1)+o(\Delta t)) \Delta t P_{(r-1, f)}(t) \\
& +(\gamma(f-1) r+o(\Delta t)) \Delta t P_{(r, f-1)}(t) \\
& +(\beta(r+1) f+o(\Delta t)) \Delta t P_{(r+1, f)}(t) \\
& +(\delta(f+1)+o(\Delta t)) \Delta t P_{(r, f+1)}(t)
\end{aligned}
$$

for $r=0,1,2 \ldots$ and $f=0,1,2 \ldots$.

Rearranging (2) and letting $\Delta t \rightarrow 0$, we obtain for $r=0,1,2 \ldots$ and $f=0,1,2 \ldots$, the state equations for this process as

$$
\begin{aligned}
P_{r, f}^{\prime}(t) & =\lim _{\Delta t \rightarrow 0} \frac{P_{r, f}(t+\Delta t)-P_{r, f}(t)}{\Delta t} \\
& =-(\beta r f+\delta f+r \alpha+r f \gamma) P_{r, f}(t)+\alpha(r-1) P_{(r-1, f)}(t) \\
& +\gamma(f-1) r P_{(r, f-1)}(t)+\beta(r+1) f P_{(r+1, f)}(t) .
\end{aligned}
$$

The doubly infinite system (3) of differential equations is not easily solved and in fact, it appears to be an open problem to obtain its closed form solution. It should be noted that a numerical solution for birth-death type systems is possible through the method of randomization. The basic method is given in [1], its application to (3) is a subject of possible further investigation to the interested reader.

The system can however, be studied by letting

$$
\phi\left(z_{1}, z_{2}, t\right)=\sum_{r=0}^{\infty} \sum_{f=0}^{\infty} P_{r, f}(t) z_{1}^{r} z_{2}^{f}
$$

be the probability generating function (p.g.f.) for this system. Then, $\phi(\cdot, \cdot, \cdot)$ can be expressed as a partial differential equation. 
In particular, using (3) in (4) gives

$$
\begin{aligned}
\sum_{r=0}^{\infty} \sum_{f=0}^{\infty} P_{r, f}^{\prime}(t) z_{1}^{r} z_{2}^{f}= & \sum_{r=0}^{\infty} \sum_{f=0}^{\infty}(-(\beta r f+\delta f+r \alpha+r f \gamma)) P_{r, f}(t) z_{1}^{r} z_{2}^{f} \\
& +\sum_{r=0}^{\infty} \sum_{f=0}^{\infty}\left(\alpha(r-1) P_{(r-1), f}(t)\right) z_{1}^{r} z_{2}^{f} \\
& +\sum_{r=0}^{\infty} \sum_{f=0}^{\infty}\left(\gamma(f-1) r P_{r,(f-1)}(t)\right) z_{1}^{r} z_{2}^{f} \\
& +\sum_{r=0}^{\infty} \sum_{f=0}^{\infty}\left(\beta(r+1) f P_{(r+1),}(t)\right) z_{1}^{r} z_{2}^{f} \\
& +\sum_{r=0}^{\infty} \sum_{f=0}^{\infty}\left(\delta(f+1) P_{r,(f+1)}(t)\right) z_{1}^{r} z_{2}^{f} .
\end{aligned}
$$

Expanding by distributing the summation through the first term in (5), we obtain

$$
\begin{aligned}
\frac{\partial \phi\left(z_{1}, z_{2}, t\right)}{\partial t}= & -\sum_{r=0}^{\infty} \sum_{f=0}^{\infty} r f \gamma P_{r, f}(t) z_{1}^{r} z_{2}^{f}-\sum_{r=0}^{\infty} \sum_{f=0}^{\infty} f \alpha P_{r, f}(t) z_{1}^{r} z_{2}^{f} \\
& -\sum_{r=0}^{\infty} \sum_{f=0}^{\infty} \delta r f P_{r, f}(t) z_{1}^{r} z_{2}^{f} \\
& +\sum_{r=0}^{\infty} \sum_{f=0}^{\infty}\left[\beta(r+1) f P_{(r+1), f}(t)\right] z_{1}^{r} z_{2}^{f} \\
& +\sum_{r=0}^{\infty} \sum_{f=0}^{\infty}\left[\delta(f+1) P_{r,(f+1)}(t)\right] z_{1}^{r} z_{2}^{f} .
\end{aligned}
$$

Recognizing partial derivatives of the pgf (4), we see that

$$
\begin{aligned}
\frac{\partial \phi\left(z_{1}, z_{2}, t\right)}{\partial t}= & \beta\left[z_{2}\left(1-z_{1}\right)-\gamma z_{1} z_{2}\left(1-z_{2}\right)\right] \frac{\partial^{2} \phi\left(z_{1}, z_{2}, t\right)}{\partial z_{2} \partial z_{1}} \\
& +\delta\left(1-z_{2}\right) \frac{\partial \phi\left(z_{1}, z_{2}, t\right)}{\partial z_{2}} \\
& -\alpha z_{1}\left(1-z_{1}\right) \frac{\partial \phi\left(z_{1}, z_{2}, t\right)}{\partial z_{1}}
\end{aligned}
$$


as the partial differential equation for the probability generating function of the system (3). From this expression, the (possible) intractibility of the system (3) is apparent.

The probability generating function (4) can be used to find the expected population sizes. In particular, differentiating (6) with respect to $z_{1}$ gives an expression for the expected prey population size as

$$
\begin{aligned}
\frac{\partial}{\partial z_{1}} \frac{\partial \phi\left(z_{1}, z_{2}, t\right)}{\partial t}= & \left(2 z_{1}-1\right) \alpha \frac{\partial \phi\left(z_{1}, z_{2}, t\right)}{\partial z_{1}} \\
& +z_{1}\left(z_{1}-1\right) \alpha \frac{\partial^{2} \phi\left(z_{1}, z_{2}, t\right)}{\partial z_{1}^{2}} \\
& +\left(1-z_{2}\right) \delta \frac{\partial^{2} \phi\left(z_{1}, z_{2}, t\right)}{\partial z_{2} \partial z_{1}} \\
& +\left(-z_{2} \beta+\left(z_{2}^{2}-z_{2}\right) \gamma\right) \frac{\partial^{2} \phi\left(z_{1}, z_{2}, t\right)}{\partial z_{2} \partial z_{1}} \\
& +\left[z_{2}\left(1-z_{1}\right) \beta+z_{1} z_{2}\left(z_{2}-1\right) \gamma\right] \frac{\partial^{2} \phi\left(z_{1}, z_{2}, t\right)}{\partial z_{2} \partial z_{2}^{2}}
\end{aligned}
$$

Substituting $z_{1}=z_{2}=1$ and recognizing expectations gives

$$
\frac{\partial}{\partial t} E(R(t))=\alpha E(R(t))-\beta E(R(t) F(t))
$$

where $E(\cdot)$ is the expectation.

Similarly, the expectation for the predators is found by differentiating (6) with respect to $z_{2}$. In particular,

$$
\begin{aligned}
\frac{\partial}{\partial z_{2}} \frac{\partial \phi\left(z_{1}, z_{2}, t\right)}{\partial t}= & z_{1}\left(z_{1}-1\right) \alpha \frac{\partial^{2} \phi\left(z_{1}, z_{2}, t\right)}{\partial z_{1} \partial z_{2}}-\delta \frac{\partial \phi\left(z_{1}, z_{2}, t\right)}{\partial z_{2}} \\
& +\left(1-z_{2}\right) \delta \frac{\partial^{2} \phi\left(z_{1}, z_{2}, t\right)}{\partial z_{2}} \\
& +\left[\left(1-z_{1}\right) \beta+\left(2 z_{1} z_{2}-z_{1}\right) \gamma\right] \frac{\partial^{2} \phi\left(z_{1}, z_{2}, t\right)}{\partial z_{2} \partial z_{1}} \\
& +\left[\left(z_{2}\left(1-z_{1}\right) \beta\right)+z_{1} z_{2}\left(z_{2}-1\right) \gamma\right] \frac{\partial^{3} \phi\left(z_{1}, z_{2}, t\right)}{\partial^{2} z_{2} \partial z_{1}} .
\end{aligned}
$$

Evaluating this expression with $z_{1}=z_{2}=1$, we obtain

$$
\frac{\partial}{\partial t} E(F(t))=\gamma E(R(t) F(t))-\delta E(F(t)) .
$$

Since $R(t)$ is a time dependent random variable, $E(R(t))$ is a time dependent deterministic quantity. The same is true for $F(t)$. Thus 
in this context, (6) and (7) are the predator-prey model obtained in (1).

\section{REFERENCES}

[1] Gross, D. and Miller, D. The Randomization Technique as a Modeling Tool and Solution Procedure for Transient Markov Processes. Operations Research. 32 (1984), 343-381.

[2] Mooney, D. D. and Swift, R. J. A Course in Mathematical Modeling. The Mathematical Association of America, Washington D.C., 1999.

[3] Tuckwell, H. C. Elementary Applications of Probability Theory. Chapman \& Hall, London, 1995.

Randall J. Swift,

Department of Mathematics,

California State Polytechnic University,

Pomona, CA 91768, USA

rjswift@csupomona.edu

Received on 25 October 2000. 